\title{
$s$-channel single top quark production and decay at next-to-next-to-leading-order in QCD
}

\author{
Ze Long Liu ${ }^{1, *}$ and Jun $\mathrm{Gao}^{2, \dagger}$ \\ ${ }^{1}$ PRISMA Cluster of Excellence \& Mainz Institute for Theoretical Physics, Johannes Gutenberg University, \\ D-55099 Mainz, Germany \\ ${ }^{2}$ INPAC, Shanghai Key Laboratory for Particle Physics and Cosmology, School of Physics and Astronomy, \\ Shanghai Jiao Tong University, Shanghai 200240, China
}

(Received 1 August 2018; published 4 October 2018)

\begin{abstract}
We report on a fully differential next-to-next-to-leading order (NNLO) calculation of $s$-channel single top (anti)quark production with a semileptonic decay at the LHC, neglecting the color correlation between the light and heavy quark lines and in the narrow width approximation. The NNLO corrections can increase the cross section by about $10 \%$ in the low transverse momentum region of the top quark and reduce scale variation uncertainty. In order to compare with experimental results without unfolding procedures, we also present theoretical predictions with fiducial cuts, including total cross sections and distributions of observables used in the experimental multivariate analysis. The NNLO corrections are found to be about $-8 \%$ for fiducial cross sections.
\end{abstract}

DOI: 10.1103/PhysRevD.98.071501

\section{INTRODUCTION}

In the standard model (SM) of particle physics, the top quark is the heaviest elementary particle. The study of top quarks is of great importance for understanding the nature of electroweak symmetry breaking and the fate of the electroweak vacuum [1-3]. There are three major modes of electroweak single top quark production at the LHC: $t$-channel, $s$-channel and $t W$-associated production. The processes are directly sensitive to the Cabbibo-KobayashiMaskawa (CKM) matrix element $V_{t b}$. $s$-channel production is of special interest though the cross section is the smallest. It is sensitive to new resonances such as $W^{\prime}$ or charged Higgs bosons involved in various models beyond the standard model (BSM) physics [4,5]. It also serves as an important background process to Higgs studies and BSM searches [6-9].

$s$-channel single top quark production was first observed by the D0 Collaboration in 2013 [10], and it was confirmed in the combined analysis by the D0 and CDF Collaborations [11] at the Fermilab Tevatron. Recently, it was also measured by the ATLAS and CMS Collaborations at the LHC with 7 and $8 \mathrm{TeV}$ data $[12,13]$. The measurements are expected to enter a precision era with increasing energy and luminosity of the (HL-)LHC.

To improve the accuracy of theoretical predictions, nextto-leading order (NLO) QCD corrections for $s$-channel

\footnotetext{
liu@uni-mainz.de

jung49@sjtu.edu.cn
}

Published by the American Physical Society under the terms of the Creative Commons Attribution 4.0 International license. Further distribution of this work must maintain attribution to the author(s) and the published article's title, journal citation, and DOI. Funded by SCOAP ${ }^{3}$. single top quark production have been calculated with and without considering the subsequent top quark decay [14-25]. In Refs. [26-28], the NLO calculations were also matched to parton shower. The soft gluon resummations were performed in Refs. [29-31]. The NLO QCD correction for $s$-channel production at the LHC is about $35 \%$, which is much larger than the estimation from scale variations at leading order (LO). To control the perturbative uncertainty, it is mandatory to calculate corrections at higher orders.

In this article, we present a next-to-next-to-leading order (NNLO) QCD calculation of $s$-channel single top (anti) quark production and decay at the LHC using the phase space slicing method. The inclusive and fully differential cross sections of a stable top (anti)quark production are obtained by neglecting the gluon exchange between light and heavy quark lines. In practice, various kinematic cuts on final states are always involved in experimental analyses to suppress large backgrounds. With the known result of top quark decay at NNLO in QCD [32], the fiducial cross sections at the LHC $13 \mathrm{TeV}$ are provided in the narrow width approximation. Distributions of various observables within the fiducial volume are also studied. These should be helpful for experimental multivariate analyses to improve the separation between signal and background.

In the following paragraphs, we outline the method used in the calculation and present numerical results on the inclusive and fiducial cross sections. Various kinematic distributions are also shown in detail.

\section{METHOD}

For $s$-channel single top (anti)quark production, QCD corrections can be separated into three categories: corrections associated with the initial state (light quark line), the final 


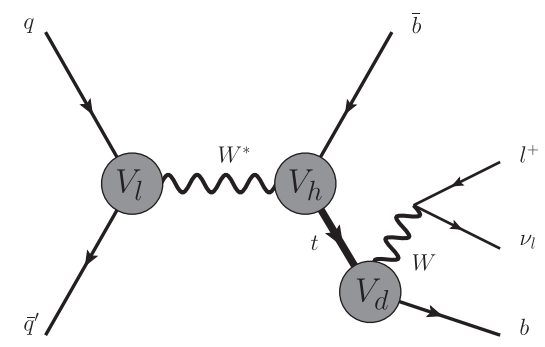

FIG. 1. Schematic diagram for $s$-channel single top quark production and decay at hadron colliders.

state (heavy quark line) and gluon exchanges between them. At NLO, the gluon exchange between the light and heavy quark lines gives no contribution due to the tracelessness of Gell-Mann matrices. At NNLO, the color factor of the diagrams with color connection between the two quark lines are suppressed by $1 / N_{c}^{2}$ compared with the corrections on the light or heavy quark lines alone [33]. Though many efforts have been devoted to calculate two-loop virtual correction in the color-connected piece of single top quark production [34,35], it is still far from complete. Here, we treat the corrections for light and heavy quark lines separately, and neglect color connections between them. In the narrow width approximation, the top (anti)quark decay is also included, of which the NNLO correction has been studied in detail in Ref. [32]. Our strategy can be summarized as in Fig. 1, where $V_{l}, V_{h}$, and $V_{d}$ denote QCD corrections from the light quark line, heavy quark line and top quark decay, respectively. All of them are separately gauge invariant and infra red (IR) safe.

To handle the IR divergences, we employ the phase space slicing method, which has been applied in NNLO QCD calculations of various processes [32,36-48]. A general method named $N$-jettiness subtraction $[43,49]$ is available to the processes with massless parton in final state. For any infrared safe observable $O$, the differential cross section can be expressed as

$$
\frac{d \sigma}{d O}=\underbrace{\int_{0}^{\tau_{\text {cut }}} d \tau \frac{d \sigma}{d \tau d O}}_{\text {unresolved }}+\underbrace{\int_{\tau_{\text {cut }}}^{\tau_{\max }} d \tau \frac{d \sigma}{d \tau d O}}_{\text {resolved }},
$$

where $\tau$ is a slicing variable. Below the cutoff $\tau_{\text {cut }}$, given $\tau_{\text {cut }}$ sufficiently small, all the radiations are unresolved, i.e., either soft or collinear to the beam or jet axes. Those contributions can be systematically factorized with softcollinear effective theory (SCET) [50-55] at leading power of $\tau_{\text {cut }}$. Progress has been made to compute the subleading power corrections [56-59].

For the light quark line, we adopt the 0 -jettiness with two beam axes as the slicing variable. For the unresolved part, the factorization formula was derived in Ref. [60]. The hard, soft and quark beam functions are available up to NNLO [61-66]. For the resolved part, the NNLO contribution is equivalent to the NLO cross section of $p p \rightarrow W^{*}+$ jet. The one-loop amplitudes of $q+\bar{q}^{\prime} \rightarrow W^{*}+g$ and $q(\bar{q})+g \rightarrow W^{*}+$ $q^{\prime}\left(\bar{q}^{\prime}\right)$ can be obtained by a nontrivial analytical continuation of the one-loop amplitudes of $e^{+} e^{-} \rightarrow q \bar{q} g[67,68]$. The dipole subtraction [69] is employed to deal with IR divergences at NLO. By setting the top quark mass $m_{t}=0$, the NNLO correction has been cross checked with result from DYNNLO [36,37].

For the heavy quark line, by neglecting the bottom quark mass and clustering all the massless partons in final state into a single jet, the slicing variable $\tau_{h}$ is defined as $\tau_{h}=m_{J}^{2} / Q^{2}$, where $m_{J}$ and $Q$ are the invariant masses of the jet and off-shell $W$ boson, respectively. In the limit of $\tau_{h} \rightarrow 0$, all QCD radiation should be soft or collinear to the bottom quark direction. The unresolved cross section can be expressed as

$$
\begin{aligned}
\left.\frac{d \sigma_{h}}{d O}\right|_{\text {unres }}= & \sum_{q, \bar{q}^{\prime}} f_{q} \otimes f_{\bar{q}^{\prime}} \otimes H_{h} \otimes S_{h} \otimes J_{q} \\
& +\mathcal{O}\left(\tau_{h, \mathrm{cut}} \ln ^{k} \tau_{h, \mathrm{cut}}\right),
\end{aligned}
$$

where $f_{q}, H_{h}, S_{h}$ and $J_{q}$ are the parton distribution function (PDF), hard function, soft function and quark jet function, respectively. The quark jet function is already known up to $\mathcal{O}\left(\alpha_{s}^{3}\right)$ [70-72]. The NNLO soft function can be obtained from Ref. [73] by boosting to the rest frame of the top quark. The hard function encodes the contribution of virtual corrections, which only depend on the dimensionless variables $x=\left(p_{b}+p_{t}\right)^{2} / m_{t}^{2}$ and $L_{t}=\ln \left(\mu / m_{t}\right)$, with $\mu$ being the renormalization scale. $p_{t}$ and $p_{b}$ denote the momenta of the top quark and bottom antiquark, respectively. In Refs. [74-77], QCD corrections to the $b \rightarrow u$ current was calculated up to $\mathcal{O}\left(\alpha_{s}^{2}\right)$ analytically. The results were expressed in terms of a set of harmonic polylogarithms (HPLs), which have a well-defined analytical continuation. Thus, we can use it to derive $H_{h}\left(x, m_{t}^{2}, \mu\right)$ by restoring the imaginary part

$$
x=\frac{\left(p_{b}+p_{t}\right)^{2}+i \varepsilon}{m_{t}^{2}-i \varepsilon}=\frac{\left(p_{b}+p_{t}\right)^{2}}{m_{t}^{2}}+i \varepsilon,
$$

with $\varepsilon$ being an infinitesimal. As a cross check, we performed analytical continuation of the matching coefficients in Refs. [75] and [76] independently and found the same results. For the resolved part, there is at least one additional hard jet due to the phase space constraint $\tau_{h}>\tau_{h, \text { cut }}$. At NNLO, this contribution can be described by the NLO corrections to $W^{*} \rightarrow \bar{b}+t+$ jet. The one-loop virtual correction can be obtained from Ref. [78] with crossing. Dipole subtraction [79] is employed to handle the IR singularities.

\section{NUMERICAL RESULTS}

The relevant parameters used in our numerical calculation are listed as follows. The top quark and $W$ boson masses are set to 172.5 and $80.385 \mathrm{GeV}$, respectively. The Fermi constant $G_{F}$ is chosen as $1.166379 \times 10^{-5} \mathrm{GeV}^{-2}$. 


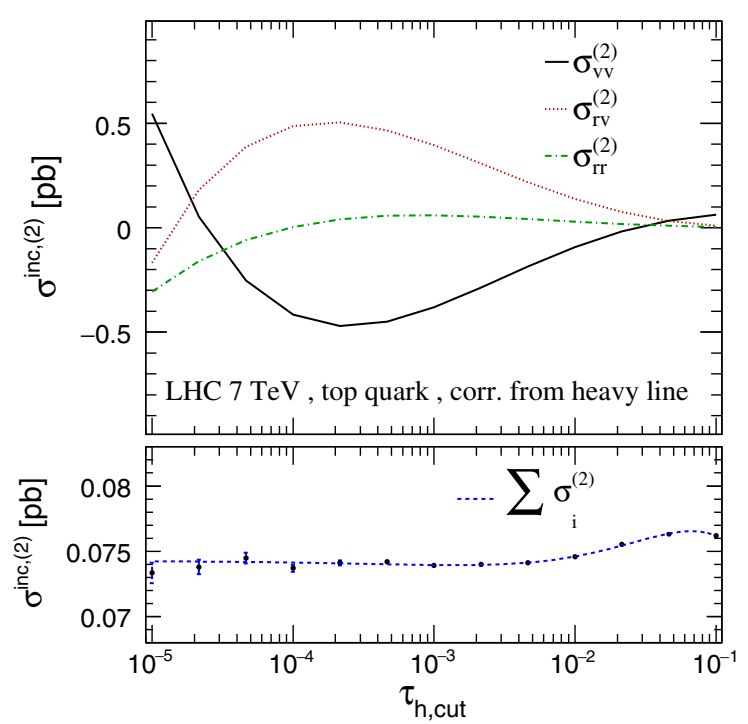

FIG. 2. Cutoff dependence of various components of NNLO corrections from heavy quark line. The lower plot shows the sum of $\sigma_{v v}^{(2)}, \sigma_{r v}^{(2)}$ and $\sigma_{r r}^{(2)}$.

The CKM matrix elements are set to $\left\{\left|V_{u d}\right|,\left|V_{u s}\right|,\left|V_{t b}\right|\right\}=$ $\{0.975,0.222,1\}$. The default values of the renormalization scale $\mu_{R}$ and factorization scale $\mu_{F}$ are chosen as $\mu_{R}=$ $\mu_{F}=m_{t}$. The scale uncertainties are calculated by varying $\mu_{R}$ and $\mu_{F}$ simultaneously by a factor of two from the default value. We use the CT14NNLO PDF set [80] with $\alpha_{s}\left(M_{Z}\right)=$ 0.118 .

Figure 2 shows three components of the NNLO corrections as a function of the cutoff $\tau_{h \text {,cut }}$ for the heavy quark line. $\sigma_{v v}^{(2)}, \sigma_{r v}^{(2)}$ and $\sigma_{r r}^{(2)}$ denote the contributions from the two-loop virtual correction, the one-loop real-virtual correction and the double real correction, respectively. The sum of them converge smoothly to $0.074 \mathrm{pb}$ as $\tau_{h, \text { cut }}$ approaching 0 . The dependence of the inclusive cross section on $\tau_{h \text {,cut }}$ are negligible for $\tau_{h \text {,cut }}$ below $10^{-3}$, as expected.

In Table I, we present the inclusive cross section of $s$-channel single top (anti)quark production at the LHC 8 and $13 \mathrm{TeV}$. Both of the NLO and NNLO corrections enhance the inclusive cross sections. The NLO corrections are typically $35 \%$. The NNLO corrections are about $7 \%$ in general, indicating a good perturbative convergence. The scale variations for the LO cross section are quite small due to the opposite trend of the $u$ and $\bar{d}$ quark PDFs from varying the factorization scale. The NNLO corrections would be underestimated by the scale variations of the NLO cross sections. Nevertheless, the scale variations are largely reduced with the NNLO corrections. At NLO, both of the corrections to $V_{l}$ and $V_{h}$ are significant. At NNLO, the corrections to $V_{l}$ are below $1 \%$, much smaller compared to the corrections to $V_{h}$ and the product of the $\mathcal{O}\left(\alpha_{s}\right)$ corrections to $V_{l}$ and $V_{h}$, which are more than 2\%. QCD corrections are similar for top quark and antiquark production. The ratio of the two cross sections are thus stable against QCD
TABLE I. Inclusive cross section for $s$-channel single top (anti) quark production at LO, NLO and NNLO at the LHC 8 and $13 \mathrm{TeV}$. The uncertainties refer to the variation by simultaneously changing the factorization and renormalization scales by a factor of two from their central value $\mu_{F}=\mu_{R}=m_{t}$.

\begin{tabular}{ccccc}
\hline \hline Inclusive & & LO & NLO & NNLO \\
\hline $8 \mathrm{TeV}$ & $\sigma(t)[\mathrm{pb}]$ & $2.498_{-0.74 \%}^{+0.17 \%}$ & $3.382_{-1.81 \%}^{+2.36 \%}$ & $3.566_{-0.78 \%}^{+0.95 \%}$ \\
& $\sigma(\bar{t})[\mathrm{pb}]$ & $1.418_{-0.12 \%}^{+0.12 \%}$ & $1.922_{-1.81 \%}^{+2.37 \%}$ & $2.029_{-0.07 \%}^{+1.07 \%}$ \\
& $\sigma(t+\bar{t})[\mathrm{pb}]$ & $3.916_{-0.15 \%}^{+0.15 \%}$ & $5.304_{-1.81 \%}^{+2.36 \%}$ & $5.595_{-0.80 \%}^{+0.99 \%}$ \\
& $\sigma(t) / \sigma(\bar{t})$ & $1.762_{-0.01 \%}^{+0.04 \%}$ & $1.760_{-0.02 \%}^{+0.00 \%}$ & $1.757_{-0.12 \%}^{+0.05 \%}$ \\
$13 \mathrm{TeV}$ & $\sigma(t)[\mathrm{pb}]$ & $4.775_{-3.50 \%}^{+2.69 \%}$ & $6.447_{-0.91 \%}^{+1.39 \%}$ & $6.778_{-0.53 \%}^{+0.76 \%}$ \\
& $\sigma(\bar{t})[\mathrm{pb}]$ & $2.998_{-3.55 \%}^{+2.69 \%}$ & $4.043_{-0.34 \%}^{+1.33 \%}$ & $4.249_{-0.48 \%}^{+0.69 \%}$ \\
& $\sigma(t+\bar{t})[\mathrm{pb}]$ & $7.772_{-3.52 \%}^{+2.69 \%}$ & $10.49_{-0.96 \%}^{+1.36 \%}$ & $11.03_{-0.51 \%}^{+0.74 \%}$ \\
& $\sigma(t) / \sigma(\bar{t})$ & $1.593_{-0.01 \%}^{+0.05 \%}$ & $1.595_{0.03 \%}^{+0.06 \%}$ & $1.595_{-0.05 \%}^{+0.07 \%}$ \\
\hline \hline
\end{tabular}

corrections, varying at the per mille level. In Table II, we show similar results for inclusive cross sections with a dynamic scale choice of $\mu_{F}=\mu_{R}=m_{W *}$. Comparing with the results in Table I, scale variations for the two scale choices are very close at all orders. Differences of the central predictions using the two scales are well within the scale variations in general. Especially, the differences at NNLO are only a few per mille indicating stabilization due to higher order corrections.

Figure 3 shows the transverse momentum distribution of top quark at the LHC $13 \mathrm{TeV}$. Both the NLO and NNLO corrections are positive and large. The ratios of NLO to LO cross sections vary from 1.2 to 1.4 over the range $0<p_{T, \text { top }}<200 \mathrm{GeV}$, and the ratios of NNLO to LO cross sections vary from 1.35 to 1.45 for the same range. In low $p_{T, \text { top }}$ region, the NNLO corrections can be as large as $10 \%$. There is no overlap between the NLO and NNLO prediction bands in most region, which again indicates the NNLO corrections would be underestimated by scale variations at NLO. The scale variations are greatly reduced going from NLO to NNLO for large $p_{T, \text { top }}$ values. In the lower panel of Fig. 3, we also present the NLO and NNLO

TABLE II. Inclusive cross section with the same setup as Table I, except for the central value of $\mu_{F, R}$ is chosen as the invariant mass of the off-shell $W$ boson.

\begin{tabular}{lcccc}
\hline \hline Inclusive & & $\mathrm{LO}$ & $\mathrm{NLO}$ & $\mathrm{NNLO}$ \\
\hline $8 \mathrm{TeV}$ & $\sigma(t)[\mathrm{pb}]$ & $2.481_{-0.42 \%}^{+0.00 \%}$ & $3.329_{-1.53 \%}^{+1.96 \%}$ & $3.550_{-0.82 \%}^{+1.03 \%}$ \\
& $\sigma(\bar{t})[\mathrm{pb}]$ & $1.409_{-0.39 \%}^{+0.00 \%}$ & $1.893_{-1.59 \%}^{+1.99 \%}$ & $2.022_{-0.91 \%}^{+0.87 \%}$ \\
& $\sigma(t+\bar{t})[\mathrm{pb}]$ & $3.890_{-0.41 \%}^{+0.00 \%}$ & $5.222_{-1.54 \%}^{+1.97 \%}$ & $5.572_{-0.85 \%}^{+0.99 \%}$ \\
& $\sigma(t) / \sigma(\bar{t})$ & $1.761_{-0.02 \%}^{+0.06 \%}$ & $1.758_{-0.03 \%}^{+0.03 \%}$ & $1.756_{-0.00 \%}^{+0.16 \%}$ \\
$13 \mathrm{TeV}$ & $\sigma(t)[\mathrm{pb}]$ & $4.836_{-2.89 \%}^{+2.21 \%}$ & $6.385_{-0.68 \%}^{+1.01 \%}$ & $6.745_{-0.40 \%}^{+0.84 \%}$ \\
& $\sigma(\bar{t})[\mathrm{pb}]$ & $3.036_{-2.22 \%}^{+2.22 \%}$ & $4.005_{-0.66 \%}^{+0.96 \%}$ & $4.235_{-0.41 \%}^{+0.67 \%}$ \\
& $\sigma(t+\bar{t})[\mathrm{pb}]$ & $7.871_{-2.91 \%}^{+2.21 \%}$ & $10.390_{-0.68 \%}^{+0.99 \%}$ & $10.980_{-0.40 \%}^{+0.77 \%}$ \\
& $\sigma(t) / \sigma(\bar{t})$ & $1.593_{-0.01 \%}^{+0.04 \%}$ & $1.594_{-0.02 \%}^{+0.04 \%}$ & $1.593_{-0.00 \%}^{+0.18 \%}$ \\
\hline \hline
\end{tabular}




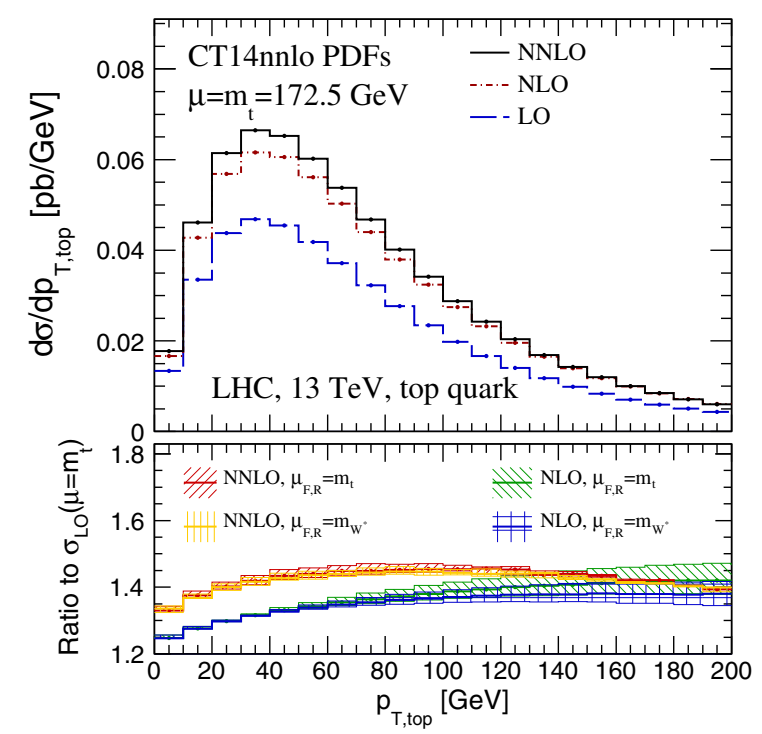

FIG. 3. Predicted transverse momentum distribution of the top quark from $s$-channel production at the LHC $13 \mathrm{TeV}$. In the lower panel, we present the distributions with $\mu_{F, R}=m_{t}$ and $\mu_{F, R}=m_{W^{*}}$, where both of the distributions with the two scale choices are divided by the LO result with $\mu_{F, R}=m_{t}$.

distributions with dynamic scale $\mu_{F, R}=m_{W^{*}}$. In order to compare the NLO and NNLO results with two differenece scale choices clearly, we divided them by the LO result with $\mu_{F, R}=m_{t}$ uniformly. It can be seen that the NNLO distributions with the two scale choices are highly consistent with each other in the whole region of $p_{T \text {,top. The }}$ NLO distribution with dynamic scale is suppressed by about $3 \%$ in large $p_{T, \text { top }}$ region, but still in the estimation of the scale uncertainties of the result with fixed scale.

In experimental analyses, top (anti)quarks are identified through their decay products e.g., semileptonic or hadronic decays. With the advantage of our fully differential calculation, we can study observables within an experimental fiducial volume. In the following calculations, we assume top quarks always decay to $b W^{+}$and use a branching ratio of 0.1086 for the leptonic decay of the $W$ boson to one family. Based on the CMS analysis [13], we choose the following basic kinematic cuts. Events with one charged lepton are selected by requiring its transverse momentum $p_{T, l}>24 \mathrm{GeV}$ and pseudorapidity $|\eta|<2.1$. Jets are clustered with anti- $k_{T}$ jet algorithm and radius $R=0.5$. Pre-selection requires jets to have $|\eta|<4.5$ and $p_{T}>$ $20 \mathrm{GeV}$. Pseudorapidity of bottom quark initiated jets are required to satisfy $|\eta|<2.4$ according to $b$-tagging algorithms [81]. Single top quark production through $s$-channel is characterized by a final state composed of one charged lepton, missing energy originating from neutrinos, and two $b$-tagged jets. One of the $b$-jets is associated with top-quark production and the other is from top-quark decay. We employ the "2-jets 2-tags" analysis [13], which requires exactly two jets, each with
TABLE III. Total cross section within the fiducial volume at the LHC $13 \mathrm{TeV}$. The NLO and NNLO QCD corrections from top quark production and decay are also listed separately.

\begin{tabular}{cccc}
\hline \hline Fiducial [pb] & LO & NLO & NNLO \\
\hline$t$ quark Total & $0.1348_{-3.4 \%}^{+2.6 \%}$ & $0.1156_{-3.0 \%}^{+3.1 \%}$ & $0.1071_{-0.8 \%}^{+2.2 \%}$ \\
Corrections from & -0.0121 & -0.0065 \\
$\quad$ top production & & & \\
Corrections from & & -0.0071 & -0.0026 \\
$\quad$ top decay & & & \\
$\bar{t}$ quark $\begin{array}{c}\text { Total } \\
\text { Corrections from } \\
\text { top production } \\
\text { Corrections from } \\
\text { top decay }\end{array}$ & $0.0907_{-3.4 \%}^{+2.5 \%}$ & $0.0745_{-3.4 \%}^{+3.6 \%}$ & $0.0663_{-1.3 \%}^{+2.5 \%}$ \\
\hline \hline
\end{tabular}

transverse momentum greater than $40 \mathrm{GeV}$, and both being $b$-tagged.

We summarize the total cross sections at LO, NLO and NNLO with the fiducial cuts at the LHC $13 \mathrm{TeV}$ in Table III. The QCD corrections from production and decay alone are also listed. In contrast to the inclusive cross sections, both the NLO and NNLO corrections are negative for fiducial cross sections. The NLO and NNLO corrections are about $-16 \%$ and $-8 \%$, respectively. QCD corrections from decay are comparable to those from production, especially for top antiquark. The scale variations are reduced with NNLO corrections.

Next, we show distributions of two observables that are key inputs to the experimental multivariate analysis. Figure 4 presents the transverse momentum distribution of the two $b$-jet system in $s$-channel single top quark production and

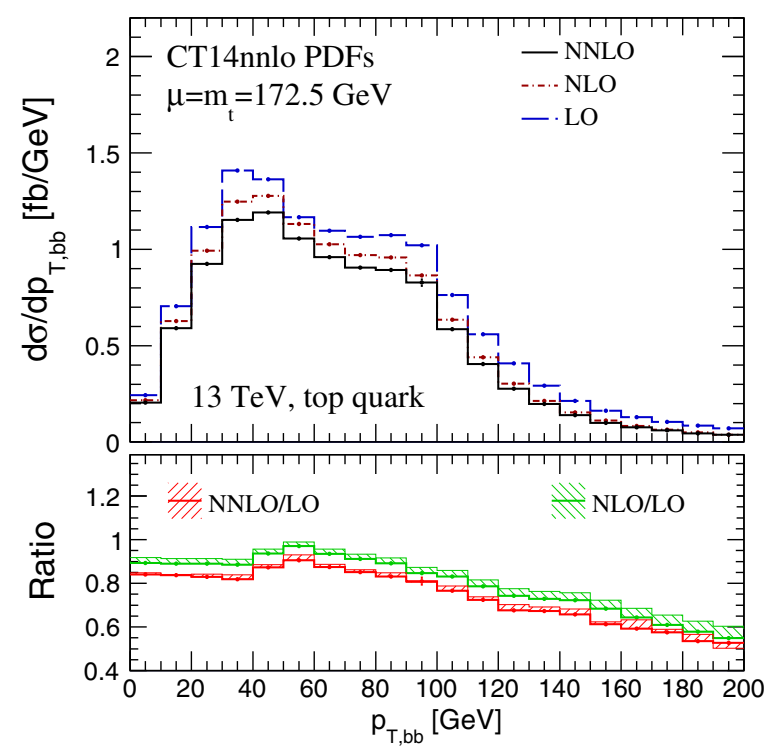

FIG. 4. Transverse momentum distribution of the two $b$-jet system from the $s$-channel single top quark production and decay at the LHC $13 \mathrm{TeV}$ with fiducial cuts. 


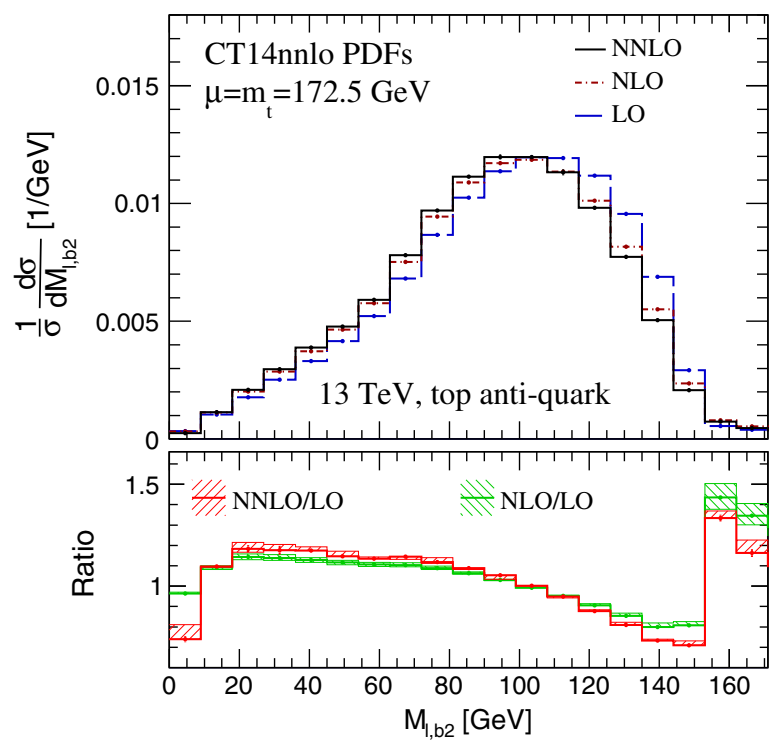

FIG. 5. Normalized distribution of invariant mass of the system composed of the charged lepton and the subleading $b$ jet in $p_{T}$, for top antiquark production and decay at the LHC $13 \mathrm{TeV}$ with fiducial cuts.

decay. The NNLO correction to the distribution is about $-10 \%$ over the range $0<p_{T, b b}<200 \mathrm{GeV}$. There is an obvious gap between the NLO and NNLO prediction bands. The scale uncertainties are reduced by NNLO corrections especially in large $p_{T, b b}$ region. Figure 5 presents the normalized distribution of the invariant mass of the system composed of the charged lepton and the subleading $b$ jet in $p_{T}$ in $s$-channel top antiquark production and decay. The distribution of $M_{l, b_{2}}$ has an endpoint around the top quark mass, as expected. The peak of the distribution is shifted to lower masses by higher order corrections. The normalized distribution show little dependence on the scale choices. The ratios of NLO and NNLO cross sections to LO ones grow rapidly when $M_{l, b_{2}}$ increases above $160 \mathrm{GeV}$, which is close to the top quark mass threshold.

\section{CONCLUSIONS}

We have presented a first NNLO QCD calculation of $s$-channel single top (anti)quark production and decay at the LHC neglecting certain subleading color contributions. The top (anti)quark spin correlation is preserved in the narrow width approximation. By considering NNLO corrections, the inclusive cross sections are enhanced by about $7 \%$ in general. The increase of cross sections at low transverse momentum of the top quark can reach above $10 \%$. Furthermore, the NNLO corrections to the total fiducial cross section are about $-8 \%$, in contrast to the inclusive case. The scale variations are reduced in general for both inclusive and fiducial cross sections. We found scale variations at NLO always underestimate the true NNLO corrections. The NNLO corrections are also significant for various kinematic distributions, including the shapes. Our results can be used to improve the measurement of cross sections of $s$-channel single top quark production, extraction of the top quark electroweak coupling and also the measurement of the top quark mass [82].

\section{ACKNOWLEDGMENTS}

We thank Hua Xing Zhu for useful communications. We are grateful to Maximilian Stahlhofen and Felix Yu for carefully reading this manuscript and precious comments. We thank Johannes Gutenberg University Mainz for the use of the High Performance Computing facility Mogon. J. G.'s work is sponsored by the Shanghai Pujiang Program. Z. L. L is supported by the Cluster of Excellence Precision Physics, Fundamental Interactions and Structure of Matter (PRISMA-EXC 1098) at JGU Mainz.
[1] G. Degrassi, S. Di Vita, J. Elias-Miro, J. R. Espinosa, G. F. Giudice, G. Isidori, and A. Strumia, J. High Energy Phys. 08 (2012) 098.

[2] S. Alekhin, A. Djouadi, and S. Moch, Phys. Lett. B 716, 214 (2012).

[3] A. Andreassen, D. Farhi, W. Frost, and M. D. Schwartz, Phys. Rev. D 95, 085011 (2017).

[4] Q.-H. Cao, J. Wudka, and C. P. Yuan, Phys. Lett. B 658, 50 (2007).

[5] T. M. P. Tait and C. P. Yuan,Phys. Rev. D 63, 014018 (2000).

[6] S. Chatrchyan et al. (CMS Collaboration), Phys. Rev. D 89, 012003 (2014).

[7] G. Aad et al. (ATLAS Collaboration), J. High Energy Phys. 01 (2015) 069.
[8] G. Aad et al. (ATLAS Collaboration), Eur. Phys. J. C 75, 165 (2015).

[9] V. Khachatryan et al. (CMS Collaboration), Eur. Phys. J. C 76, 237 (2016).

[10] V. M. Abazov et al. (D0 Collaboration), Phys. Lett. B 726, 656 (2013).

[11] T. A. Aaltonen et al. (CDF, D0 Collaboration), Phys. Rev. Lett. 112, 231803 (2014).

[12] G. Aad et al. (ATLAS Collaboration), Phys. Lett. B 756, 228 (2016).

[13] V. Khachatryan et al. (CMS Collaboration), J. High Energy Phys. 09 (2016) 027.

[14] G. Bordes and B. van Eijk, Nucl. Phys. B435, 23 (1995).

[15] M. C. Smith and S. Willenbrock, Phys. Rev. D 54, 6696 (1996). 
[16] S. Zhu, Phys. Lett. B 524, 283 (2002); 537, 351(E) (2002).

[17] B. W. Harris, E. Laenen, L. Phaf, Z. Sullivan, and S. Weinzierl, Phys. Rev. D 66, 054024 (2002).

[18] Z. Sullivan, Phys. Rev. D 70, 114012 (2004).

[19] J. M. Campbell, R. K. Ellis, and F. Tramontano, Phys. Rev. D 70, 094012 (2004).

[20] Q.-H. Cao and C. P. Yuan, Phys. Rev. D 71, 054022 (2005).

[21] Q.-H. Cao, R. Schwienhorst, and C. P. Yuan, Phys. Rev. D 71, 054023 (2005).

[22] Q.-H. Cao, R. Schwienhorst, J. A. Benitez, R. Brock, and C. P. Yuan, Phys. Rev. D 72, 094027 (2005).

[23] Q.-H. Cao, arXiv:0801.1539.

[24] J. M. Campbell, R. Frederix, F. Maltoni, and F. Tramontano, J. High Energy Phys. 10 (2009) 042.

[25] S. Heim, Q.-H. Cao, R. Schwienhorst, and C. P. Yuan, Phys. Rev. D 81, 034005 (2010).

[26] S. Frixione, E. Laenen, P. Motylinski, and B. R. Webber, J. High Energy Phys. 03 (2006) 092.

[27] S. Frixione, E. Laenen, P. Motylinski, B. R. Webber, and C. D. White, J. High Energy Phys. 07 (2008) 029.

[28] S. Alioli, P. Nason, C. Oleari, and E. Re, J. High Energy Phys. 09 (2009) 111; 02 (2010) 011(E).

[29] N. Kidonakis, Phys. Rev. D 81, 054028 (2010).

[30] H. X. Zhu, C. S. Li, J. Wang, and J. J. Zhang, J. High Energy Phys. 02 (2011) 099.

[31] N. Kidonakis, Phys. Part. Nucl. 45, 714 (2014).

[32] J. Gao, C. S. Li, and H. X. Zhu, Phys. Rev. Lett. 110, 042001 (2013).

[33] M. Brucherseifer, F. Caola, and K. Melnikov, Phys. Lett. B 736, 58 (2014).

[34] M. Assadsolimani, P. Kant, B. Tausk, and P. Uwer, Phys. Rev. D 90, 114024 (2014).

[35] C. Meyer, J. High Energy Phys. 04 (2017) 006.

[36] S. Catani and M. Grazzini, Phys. Rev. Lett. 98, 222002 (2007).

[37] S. Catani, L. Cieri, G. Ferrera, D. de Florian, and M. Grazzini, Phys. Rev. Lett. 103, 082001 (2009).

[38] S. Catani, L. Cieri, D. de Florian, G. Ferrera, and M. Grazzini, Phys. Rev. Lett. 108, 072001 (2012); 117, 089901(E) (2016).

[39] F. Cascioli, T. Gehrmann, M. Grazzini, S. Kallweit, P. Maierhöfer, A. von Manteuffel, S. Pozzorini, D. Rathlev, L. Tancredi, and E. Weihs, Phys. Lett. B 735, 311 (2014).

[40] T. Gehrmann, M. Grazzini, S. Kallweit, P. Maierhöfer, A. von Manteuffel, S. Pozzorini, D. Rathlev, and L. Tancredi, Phys. Rev. Lett. 113, 212001 (2014).

[41] J. Gao and H. X. Zhu, Phys. Rev. Lett. 113, 262001 (2014).

[42] M. Grazzini, S. Kallweit, and D. Rathlev, J. High Energy Phys. 07 (2015) 085.

[43] R. Boughezal, C. Focke, X. Liu, and F. Petriello, Phys. Rev. Lett. 115, 062002 (2015).

[44] R. Boughezal, C. Focke, W. Giele, X. Liu, and F. Petriello, Phys. Lett. B 748, 5 (2015).

[45] R. Boughezal, J. M. Campbell, R. K. Ellis, C. Focke, W. T. Giele, X. Liu, and F. Petriello, Phys. Rev. Lett. 116, 152001 (2016).

[46] E. L. Berger, J. Gao, C. P. Yuan, and H. X. Zhu, Phys. Rev. D 94, 071501 (2016).

[47] E. L. Berger, J. Gao, and H. X. Zhu, J. High Energy Phys. 11 (2017) 158.

[48] E. L. Berger, J. Gao, C. S. Li, Z. L. Liu, and H. X. Zhu, Phys. Rev. Lett. 116, 212002 (2016).

[49] J. Gaunt, M. Stahlhofen, F. J. Tackmann, and J. R. Walsh, J. High Energy Phys. 09 (2015) 058.
[50] C. W. Bauer, S. Fleming, and M. E. Luke, Phys. Rev. D 63, 014006 (2000).

[51] C. W. Bauer, S. Fleming, D. Pirjol, and I. W. Stewart, Phys. Rev. D 63, 114020 (2001).

[52] C. W. Bauer and I. W. Stewart, Phys. Lett. B 516, 134 (2001).

[53] C. W. Bauer, D. Pirjol, and I. W. Stewart, Phys. Rev. D 65, 054022 (2002).

[54] C. W. Bauer, S. Fleming, D. Pirjol, I. Z. Rothstein, and I. W. Stewart, Phys. Rev. D 66, 014017 (2002).

[55] M. Beneke, A. P. Chapovsky, M. Diehl, and T. Feldmann, Nucl. Phys. B643, 431 (2002).

[56] I. Moult, L. Rothen, I. W. Stewart, F. J. Tackmann, and H. X. Zhu, Phys. Rev. D 95, 074023 (2017).

[57] R. Boughezal, X. Liu, and F. Petriello, J. High Energy Phys. 03 (2017) 160.

[58] I. Moult, L. Rothen, I. W. Stewart, F. J. Tackmann, and H. X. Zhu, Phys. Rev. D 97, 014013 (2018).

[59] R. Boughezal, A. Isgrò, and F. Petriello, Phys. Rev. D 97, 076006 (2018).

[60] I. W. Stewart, F. J. Tackmann, and W. J. Waalewijn, Phys. Rev. D 81, 094035 (2010).

[61] A. Idilbi, X.-d. Ji, and F. Yuan, Nucl. Phys. B753, 42 (2006).

[62] T. Becher, M. Neubert, and B. D. Pecjak, J. High Energy Phys. 01 (2007) 076.

[63] R. Kelley, M. D. Schwartz, R. M. Schabinger, and H. X. Zhu, Phys. Rev. D 84, 045022 (2011).

[64] P. F. Monni, T. Gehrmann, and G. Luisoni, J. High Energy Phys. 08 (2011) 010.

[65] J. R. Gaunt, M. Stahlhofen, and F. J. Tackmann, J. High Energy Phys. 04 (2014) 113.

[66] J. Gaunt, M. Stahlhofen, and F. J. Tackmann, J. High Energy Phys. 08 (2014) 020.

[67] L. W. Garland, T. Gehrmann, E. W. N. Glover, A. Koukoutsakis, and E. Remiddi, Nucl. Phys. B642, 227 (2002).

[68] T. Gehrmann and E. Remiddi, Nucl. Phys. B640, 379 (2002).

[69] S. Catani and M. H. Seymour, Nucl. Phys. B485, 291 (1997); B510, 503(E) (1998).

[70] T. Becher and M. Neubert, Phys. Lett. B 637, 251 (2006).

[71] R. Brüser, Z. L. Liu, and M. Stahlhofen, Phys. Rev. Lett. 121, 072003 (2018).

[72] P. Banerjee, P. K. Dhani, and V. Ravindran, arXiv:1805 .02637.

[73] T. Becher and M. Neubert, Phys. Lett. B 633, 739 (2006).

[74] R. Bonciani and A. Ferroglia, J. High Energy Phys. 11 (2008) 065.

[75] H. M. Asatrian, C. Greub, and B. D. Pecjak, Phys. Rev. D 78, 114028 (2008).

[76] M. Beneke, T. Huber, and X. Q. Li, Nucl. Phys. B811, 77 (2009).

[77] G. Bell, Nucl. Phys. B812, 264 (2009).

[78] J. M. Campbell and F. Tramontano, Nucl. Phys. B726, 109 (2005).

[79] S. Catani, S. Dittmaier, M. H. Seymour, and Z. Trocsanyi, Nucl. Phys. B627, 189 (2002).

[80] S. Dulat, T.-J. Hou, J. Gao, M. Guzzi, J. Huston, P. Nadolsky, J. Pumplin, C. Schmidt, D. Stump, and C. P. Yuan, Phys. Rev. D 93, 033006 (2016).

[81] S. Chatrchyan et al. (CMS Collaboration), J. Instrum. 8, P04013 (2013).

[82] S. Alekhin, S. Moch, and S. Thier, Phys. Lett. B 763, 341 (2016). 